\title{
Elaboração de um protocolo de assistência de enfermagem ao paciente hematológico:
} relato de experiência

\section{Elaboration of a nursing care protocol for hematological patients: an experience report}

\author{
Elaboración de un protocolo de atención de enfermería al paciente hematológico: \\ relato de experiencia
}

\section{Rita Paiva Pereira Honório', Joselany Afio Caetano"}

\author{
Enfermeira; Especialista em Enfermagem Médico-Cirúrgica; Especialista em Enfermagem Oncológica; Gerente do Serviço de Educação \\ Continuada do Hospital Universitário Walter Cantídio/UFC; Mestranda do Programa de Pós-Graduação da Universidade Federal do Ceará. \\ Fortaleza/CE. Email: enfa_rita@yahoo.com.br. \\ "Enfermeira; Doutora em Enfermagem; Docente do Departamento de Enfermagem da FFOE/UFC. Email: joselany@ufc.br.
}

\section{RESUMO}

Este artigo relata a experiência do grupo de enfermeiras da unidade de internação de hematologia do Hospital Universitário Walter Cantídio (HUWC), na cidade de Fortaleza-CE, Brasil, diante da elaboração de um protocolo de assistência a pacientes com alterações hematológicas. Por meio de oficinas de trabalho, foram elaborados pelo grupo quatro procedimentos operacionais padrão que buscavam descrever os seguintes aspectos do cuidado de enfermagem: admissão do paciente; punção, heparinização e curativo do cateter totalmente implantado; administração de quimioterápicos e conduta em extravasamento; descarte de resíduos citotóxicos. No decorrer do trabalho identificaram-se as seguintes necessidades: adequar o dimensionamento de pessoal e o número de equipamentos que possibilitem a administração de quimioterápicos com segurança; elaborar manual de orientação de alta dos pacientes; buscar novos pontos críticos dos processos de trabalho e rever periodicamente os protocolos já existentes; buscar parcerias para a elaboração dos protocolos de descarte de resíduos citotóxicos e de extravasamento de quimioterápicos. Segundo acredita o grupo, a utilização de protocolos proporciona uma prática mais qualificada e uma assistência cada vez mais eficaz e humanizada ao paciente.

Descritores: Hematologia; Cuidados de Enfermagem; Avaliação em Enfermagem.

\section{ABSTRACT}

This article reports on the experience of the group of nurses working at the hematology hospitalization unit of the University Hospital Walter Cantídio (HUWC), located in Fortaleza-CE, Brazil, in view of the elaboration of a care protocol for patients with hematological alterations. Using workshops, the group elaborated four standard operational procedures that attempted to describe the following aspects of nursing care: patient admission; puncture, heparinization and dressing of the fully implanted catheter; administration of chemotherapeutic agents and conduct in case of extravasation; discarding of cytotoxic residues. During the work, the following needs were identified: adapting the staff dimensioning and the number of equipments that permit safe chemotherapeutic agent administration; elaborating an advisory manual for the patients' discharge; searching for new critical points in the work processes and periodically reconsidering existing protocols; seeking partnerships to elaborate discarding protocols for cytotoxic residue and chemotherapeutic agent extravasations. The group believes that the use of protocols offers a more qualified practice and increasingly more effective and humanized care delivery to patients.

Descriptors: Hematology; Nursing Care; Nursing Assessment.

\section{RESUMEN}

Este artículo relata la experiencia del grupo de enfermeras de la unidad de internación de hematología del Hospital Universitario Walter Cantídio (HUWC), en la ciudad de Fortaleza-CE, Brasil, ante la elaboración de un protocolo de atención a pacientes con alteraciones hematológicas. Mediante oficinas de trabajo, el grupo elaboro cuatro procedimientos operacionales estándares que buscaban describir los siguientes aspectos del cuidado de enfermería: admisión del paciente; punción, heparinización y curativo del catéter totalmente implantado; administración de quimioterápicos y conducta en transborde; descarte de residuos citotóxicos. Durante el trabajo, fueron identificadas las siguientes necesidades: adecuar el dimensionamiento de personal y el número de equipos que posibiliten la administración de quimioterápicos con seguridad; elaborar manual de orientación de alta de los pacientes; buscar nuevos puntos críticos de los procesos de trabajo y rever periódicamente los protocolos ya existentes; buscar parejas para la elaboración de los protocolos de descarte de residuos citotóxicos y de transborde de quimioterápicos. Según cree el grupo, la utilización de protocolos proporciona una práctica más cualificada y una atención cada vez más eficaz y humanizada al paciente.

Descriptors: Hematología; Cuidados de Enfermería; Protocolos de Enfermería. 


\section{NTRODUÇÃO}

O cuidado de pacientes com alterações hematológicas pode ser bastante desafiador para as enfermeiras. Este desafio justifica-se tanto por estes apresentarem distúrbios sangüíneos muito significativos, a exemplo de pancitopenia, granulocitose e vários distúrbios hemorrágicos e de coagulação, como por exigirem um cuidado meticuloso no tratamento para evitar a deterioração e as complicações a ele inerentes ${ }^{(1)}$.

A exemplo de outros pacientes, o portador de alterações hematológicas requer uma assistência de enfermagem especializada na qual a equipe o veja nas dimensões bio-psico-social. Desse modo, ele se transforma no centro de suas intervenções, com vistas à sua adaptação e ao autocuidado. Para atingir estes objetivos, torna-se evidente a necessidade de que os enfermeiros conheçam profundamente as características, os sinais e sintomas, tipos de tratamentos, efeitos colaterais e os cuidados de enfermagem que podem ser prestados, pois os enfermeiros, como membros da equipe de saúde, assumem função vital na recuperação do paciente ${ }^{(2)}$.

Para garantir a confiabilidade à assistência de enfermagem a pacientes com distúrbios hematológicos, por meio de procedimentos seguros, baseados em ações as mais científicas possíveis, é imprescindível a construção de protocolos de assistência. Por protocolos de assistência entende-se aqui um conjunto de dados que permitem direcionar - trabalho e registrar oficialmente os cuidados executados na resolução ou prevenção de um problema $^{(3)}$.

Diante destas exigências, este artigo tem o objetivo de relatar a experiência de um grupo de enfermeiras de uma unidade de hematologia na elaboração de um protocolo de assistência aos pacientes deste serviço.

Trata-se de uma proposta de padronização de procedimentos feitos pela equipe de enfermagem para auxiliar paciente com alterações hematológicas, que apresentam também distúrbios emocionais significativos ligados a ameaças de alterações na imagem corporal e na auto-imagem, perda de identidade, sentimentos de angústia, medo, depressão ou raiva, mudanças de papéis, isolamento social, dependência física e psicológica ${ }^{(4)}$.

Além dos distúrbios emocionais os clientes hematológicos podem apresentar sinais e sintomas variados, decorrentes de alterações em diversos sistemas orgânicos, pois as modificações presentes na crase sangüínea geram distúrbios na oxigenação, coagulação e sistema de defesa do organismo, provocando muitas vezes fraqueza, debilidade física, emagrecimento, febre, lesões ulcerativas de pele e mucosas, parestesias, dores, sangramentos e infecções ${ }^{(4)}$.

\section{CONTEXTUALI ZANDO A EXPERI ÊNCI A}

O Hospital Universitário Walter Cantídio é órgão suplementar da Universidade Federal do Ceará. Possui 17 leitos para hematologia, nos quais a média de internação é em torno de $90 \%$. Em virtude da complexidade do tratamento e das intercorrências inerentes às patologias, a reinternação é freqüente.

Para a elaboração dos protocolos, o grupo optou por oficinas de trabalho, mediante utilização da técnica de brainstorming ${ }^{(5)}$, onde inicialmente listaram todos os procedimentos de enfermagem para pacientes com alterações hematológicas. Para a seleção dos procedimentos, foram consideradas as dificuldades técnicas e de memorização da tarefa, a possibilidade de ocorrência de iatrogenias durante o processo de sua execução e a observação do processo de trabalho de toda a equipe de enfermagem, como: o preparo para executar a tarefa, a técnica utilizada, a abordagem, os recursos e o tipo de registro.

Após discussão, o grupo abordou os seguintes aspectos do cuidado, denominados protocolos: admissão do paciente; punção, heparinização e curativo do cateter totalmente implantado; administração de quimioterápicos e conduta em extravasamento; descarte de resíduos citotóxicos e assistência ao paciente imunodeprimido.

Numa primeira oficina as enfermeiras do estudo dividiram-se em duplas, formando quatro grupos. Cada dupla ficou responsável pela elaboração de duas rotinas. Foram agendadas oficinas quinzenais para discussão, análise e finalização de cada rotina, passo a passo, num total de 12 encontros.

$\mathrm{Na}$ segunda oficina os grupos compareceram com o esboço das rotinas. Estas foram divididas novamente entre o grupo e a cada dupla coube analisar mais duas, além das que foram por si elaboradas. Quanto às dúvidas, foram lidas em voz alta e consensuadas em grupo, tendo como suporte teórico a literatura vigente mais atualizada sobre a rotina do procedimento em construção e a Classificação das Intervenções de Enfermagem ( $\mathrm{NIC}$ ).

Intervenções de enfermagem são definidas como qualquer tratamento, baseado no julgamento clínico e conhecimento, realizado por uma enfermeira para aumentar resultados obtidos pelo paciente/cliente ${ }^{(6)}$.

Em sua última edição( ${ }^{(6)}$, a Classificação de Intervenções de Enfermagem (NIC) apresenta 486 intervenções e mais de 12.000 atividades agrupadas em 30 classes e sete domínios.

Em seguida, as rotinas foram montadas com base em um modelo de check-list onde se abordam os passos construtivos de um procedimento operacional padrão $(\mathrm{POP})^{(7)}$. 


\section{Cuidados de enfermagem durante a admissão do paciente na unidade de internação}

As intervenções de enfermagem neste momento contemplam os cuidados na admissão, controle do ambiente, redução da ansiedade, controle da nutrição, toque, controle de suprimentos, supervisão, supervisão: segurança, orientação quanto ao sistema de saúde, troca de informações sobre cuidados de saúde, educação para a saúde, avaliação da saúde, identificação de risco: genético, presença, ouvir ativamente, proteção dos direitos do paciente ${ }^{(6)}$.

A equipe de saúde deve controlar o ambiente que circunda o paciente visando a um benefício terapêutico sensorial e psicológico ${ }^{(6)}$. Neste primeiro contato entre o paciente e a equipe, o acolhimento é o momento mais importante para o estabelecimento de uma relação de confiança e cumplicidade. Existem três fatores importantíssimos para o sucesso desta tarefa, quais sejam: atenção específica; cordialidade da equipe e resolução de problemas ${ }^{(8)}$.

É fundamental que o cliente compreenda o processo de cuidado, pois, desse modo, poderá identificar sinais e sintomas da doença, maneira de minimizar riscos, formas de prevenir complicações e medidas de promoção da saúde.

\section{Cuidados de enfermagem com $o$ cateter totalmente implantado desde a punção, heparinização e curativo}

Tendo em vista o longo tempo de tratamento do paciente com alterações hematológicas e o tipo de drogas citotóxicas das quais ele necessita fazer uso, sua rede venosa torna-se de difícil acesso e extremamente frágil às punções venosas periféricas.

De acordo com a média de tempo do tratamento a que o paciente será submetido, torna-se mais seguro para ele o cateter totalmente implantado. Este cateter tem boa aceitação por não requerer cuidados domiciliares e apresentar mínima interferência na auto-imagem. São dispositivos de borracha siliconizada cuja extremidade distal se acopla a uma câmera puncionável, que deve permanecer sob a pele, embutida em uma loja no tecido subcutâneo da região torácica, sobre uma protuberância óssea $^{(9)}$.

O treinamento dos profissionais de enfermagem que manipulam diariamente estes cateteres, e a existência de protocolos rigorosos de cuidados, têm possibilitado a redução nos riscos de complicações infecciosas $^{(10)}$.

Em relação ao protocolo de heparinização, um estudo de coorte avaliou a incidência de trombose relacionada aos cateteres tunelizados em pacientes que receberam a infusão de $5 \mathrm{ml}$ da solução salina com heparina $10 \mathrm{U} / \mathrm{ml}$ e de $10 \mathrm{ml}$ da solução salina com heparina $100 \mathrm{U} / \mathrm{ml}$. Segundo os resultados demonstraram, não houve diferença estatisticamente significante entre os dois protocolos ${ }^{(11)}$.
As diretrizes da CDC para o curativo de cateteres tunelizados orientam que pode ser tanto com gaze estéril e esparadrapo quanto com películas de poliuretano e que deve ser refeito quando estiver úmido, solto ou sujo. Na anti-sepsia da pele há preferência pela solução clorexidina a $2 \%$, embora o PVPI alcoólico e aquoso ou o álcool a 70\% também possam ser utilizados(12). Quando comparados os diferentes tipos de cobertura para o curativo de cateter de Hickman, não foi encontrada, nos estudos, diferença estatisticamente significante na incidência de infecção relacionada ao cateter ${ }^{(13)}$.

As intervenções apontadas foram: inserção endovenosa, manutenção de dispositivo para acesso venoso, terapia endovenosa, controle de alergia, controle de infecção, controle de choque, ensino: medicação prescrita, transcrição de prescrições, apoio ao médico, prescrição de medicamentos, documentação, administração de medicamentos: endovenosa, controle de medicamentos ${ }^{(6)}$.

\section{Cuidados de enfermagem na administração de quimioterápicos por via endovenosa e conduta em extravasamento}

Entre as modalidades de tratamento oncológico, a quimioterapia é a que possui maior incidência de cura e a que mais aumenta a sobrevida dos portadores de câncer(14). É uma modalidade de tratamento sistêmica na qual os agentes antineoplásicos são tóxicos a qualquer tecido de rápida proliferação, normal ou canceroso, caracterizado por uma alta atividade mitótica e ciclo celular curto e, deste modo, tem como conseqüência o aparecimento de efeitos colaterais ${ }^{(15)}$.

$\mathrm{Na}$ execução do protocolo foram realizadas as seguintes intervenções: promoção do envolvimento familiar, controle de infecção, controle da quimioterapia, assistência em exames, orientação para a realidade, controle de medicamentos, controle de vômito, ensino: procedimento e tratamento, ensino: medicação prescrita, transcrição de prescrições, prescrição de medicamentos, documentação, administração de medicamentos: endovenosa, controle de medicamentos, controle da nutrição, controle de líquidos/eletrólitos, monitorização de eletrólitos, cuidados com lesões, proteção contra infecção ${ }^{(6)}$.

Três cuidados são indispensáveis na administração de quimioterápicos. Em primeiro lugar, a atenção rigorosa à prescrição médica, ao nome da droga, à dose, à via de aplicação e à identificação completa do paciente. Em segundo, os rigores quanto à assepsia e às medidas de proteção ao paciente e ao operador e, em terceiro, o conhecimento da droga nos seus aspectos de diluição, conservação, estabilidade, incompatibilidades e fotossensibilidade. O National Institute for Ocupational Safety and Heath $(\mathrm{NIOSH})^{(9)}$ recomenda se utilizar de luvas duplas, 
avental impermeável e óculos, e não desconectar os equipos dos frascos contendo quimioterapia durante a infusão do quimioterápico.

Podem ocorrer, as seguintes toxicidades: hematológica, hepática, cardiológica, dermatológica, reprodutiva, renal e neurológica. As reações cutâneas mais graves devem-se ao extravasamento de fármacos vesicantes no tecido vizinho à veia puncionada, com irritação severa, formação de vesículas e destruição tecidual ${ }^{(9,15)}$.

As drogas irritantes provocam reação menos intensa do que as vesicantes, quando extravasadas (dor e queimação sem necrose tecidual ou formação de vesículas), porém, mesmo adequadamente infundidas, podem ocasionar dor e reação inflamatória no local de punção e ao longo da veia utilizada $^{(9,15)}$.

O extravasamento de quimioterápicos é a infiltração acidental da droga no tecido subcutâneo circunjacente e seus efeitos tóxicos locais variam, podendo causar dor, necrose tissular ou descamação do tecido. O potencial vesicante de uma droga, o volume extravasado, o sítio de infiltração e o tempo de exposição à droga serão fatores decisivos para determinar a extensão da lesão.

É importante reconhecer e tratar precocemente o extravasamento dos citotóxicos reduzindo, ao máximo, os danos teciduais. Para isto é preciso monitorar sinais de infiltração e flebite no local de infusão; parar a infusão do citotóxico imediatamente; aspirar a medicação residual e elevar o membro e aplicar compressa de acordo com a indicação específica ao citotóxico que extravasou; orientar o cliente a manter o membro elevado por 48 horas e notificar a ocorrência ao médico responsável e registrar no prontuário do paciente.

Para náusea e vômito, o enfermeiro deve atuar de maneira que forneça os antieméticos em quantidade suficiente no período crítico e assegure que o paciente saiba utilizá-lo. Além disso, orientá-lo acerca das refeições que deverão ser frias, com alimentos livres de gorduras e acompanhados de refrigerantes, pois são mais toleradas que os alimentos quentes, devido ao odor ${ }^{(16)}$.

\section{Cuidados de enfermagem no descarte de resíduos citotóxicos}

De acordo com a Norma Regulamentadora (NR32) cuja finalidade é estabelecer as diretrizes básicas para a implementação de medidas de proteção e segurança à saúde dos trabalhadores dos serviços de saúde, a instituição deve assegurar capacitação em biossegurança aos seus funcionários, bem como fornecer equipamentos de proteção individual específico. As normas regulamentadoras devem constar em um manual de procedimentos de quimioterapia e este deve estar disponível aos trabalhadores e à fiscalização do trabalho.
Pesquisas têm demonstrado fortes evidências de risco no manuseio de quimioterápicos e, portanto, a necessidade de cuidados especiais de proteção tanto do paciente quanto dos manipuladores destas drogas. O risco de exposição ocorre em qualquer fase, desde o preparo, até a administração e o descarte dos quimioterápicos. Da mesma forma, a manipulação de urina, fezes e demais fluidos corpóreos durante a aplicação e nas primeiras 48 horas após também oferece risco de exposição( ${ }^{(9)}$.

Para minimizar a contaminação por resíduos citotóxicos as principais atividades são: acondicionar frascos e seringas com os quimioterápicos, a serem administrados, em saco plástico fechado; utilizar os EPIs no manuseio de secreção e excretas; descartar agulhas e seringas em recipientes apropriados (descartex); manusear roupa de cama, camisolas e pijamas contaminados com luva de procedimento; descartar frascos de soro e equipos em saco plástico fechado, depositado em lixo e devidamente identificado como material contaminante.

\section{CONCLUSÕES}

Em face do uso de antineoplásicos que produzem efeitos colaterais esperados, as intervenções estavam centradas para esta terapêutica, porém em toda a assistência as intervenções de natureza educativa também estavam presentes, visando a informação do paciente sobre o processo da doença e do tratamento e sua participação no autocuidado.

Paciente com alterações hematológicas apresenta agravos ao sistema imunológico que advêm tanto da própria patologia quanto do regime terapêutico. Nesta fase crítica do tratamento, a atuação da equipe de enfermagem deverá visar a prevenção e a deteç̧ão precoce das complicações, pontos fundamentais da assistência com vistas a garantir a sobrevida do paciente.

Em virtude de diversos fatores, o risco para infecção está presente, principalmente devido às alterações advindas da doença hematológica (imunossupressão, leucopenia, anemia), bem como às conseqüências de uma doença que exige tempo de internação prolongado, utilização de procedimentos invasivos e tratamento com agentes medicamentosos (antibióticos e quimioterápicos). Se houver injúria e lesão de continuidade da pele, associadas às alterações hematológicas, aumentam os riscos para as infecções ${ }^{(4)}$.

Os pacientes hematológicos apresentam problemas específicos. Em faces destesm exige-se um plano de cuidados próprios para este tipo de paciente. Dessa forma, cabe à enfermagem utilizar recursos possíveis de aprimorar a assistência prestada a esses pacientes, com implementação de intervenções de enfermagem precisa que possibilite a melhoria da qualidade dos resultados esperados. Portanto, é indispensável o preparo da enfermeira, 
bom como dos familiares que participam do processo de assistência ao portador de alterações hematológicas. Com esta finalidade, enfatiza-se a necessidade da realização de estudos para a construção de protocolos neste âmbito do cuidado.

\section{CONSI DERAÇÕES FI NAIS}

Atualmente, a padronização é considerada a mais fundamental das ferramentas gerenciais. No entanto, conforme a experiência tem demonstrado, a situação brasileira no tocante à padronização não é boa: falta literatura e faltam educação e treinamento das pessoas que ocupam cargos de chefia. Nas raras empresas que se consideram padronizadas, este assunto tem sido relegado aos técnicos, apesar de ser um encargo essencialmente gerencial. Contudo as pessoas que ocupam cargos gerenciais precisam entender que a padronização é o caminho mais seguro para a produtividade e competitividade em nível internacional, e constitui uma das bases sobre as quais se assenta o moderno gerenciamento.

Como observado no cotidiano, a vivência em elaborar protocolos por um grupo de enfermeiros que trabalham com pacientes hematológicos proporciona crescimento para a equipe e para cada profissional, com repercussões na melhoria da qualidade da assistência de enfermagem. Tais resultados são evidenciados no decorrer do trabalho, sobretudo ao se perceber a necessidade de pontuar algumas exigências pertinentes à validação e conseqüente implantação dos processos. Entre estes, mencionamse: adequar o dimensionamento de pessoal e o número de equipamentos que possibilitem a administração de quimioterápicos com segurança; elaborar manual de orientação de alta dos pacientes; buscar novos pontos críticos dos processos de trabalho e rever periodicamente os protocolos já existentes; buscar parcerias para a elaboração dos protocolos de descarte de resíduos citotóxicos e de extravasamento de quimioterápicos.

Diante dos distúrbios hematológicos, a confecção de protocolos em equipe permite que cada profissional expresse seus conhecimentos e suas experiências, seguindo-se discussões geradoras de consenso indutor de condutas unificadas e conseqüentemente, de uma melhor qualidade de assistência. É preciso, porém, ter em mente que a jornada pela qualidade dos serviços é uma caminhada sem linha de chegada. Constitui-se de etapas, as quais, vencidas, injetam mais energia e disposição a percorrê-las. Ao se elaborar este trabalho espera-se que ele possa servir de exemplo e subsídio a diferentes investidas do enfermeiro à efetiva padronização dos processos de trabalho da equipe sob sua coordenação e, ao mesmo tempo, estimule os profissionais com vistas a reforçar a credibilidade por meio de evidências da viabilidade da aplicação da gestão pela qualidade.

\section{REFERÊNCI AS}

1. Smeltzer SC, Bare BG. Brunner \& Suddarth. Histórico e tratamento de pacientes com distúrbios hematológicos. In: Smeltzer SC, Bare BG. Brunner \& Suddarth. Tratado de enfermagem médico-cirurgica. 10th ed. Rio de Janeiro: Guanabara Koogan; 2005. p. 918-991.

2. Rogers BB. Overview of non-Hodgkin's lymphoma. Semin Oncol Nurs. 2006;22(2):67-72.

3. Barros SKS, Anami EHT, Moraes MP. A elaboração de um protocolo para prevenção de úlcera de pressão por enfermeiros de um hospital de ensino. Revista Nursing. 2003;63(6): 29-32.

4. Jesus CAC, Carvalho EC. Diagnósticos de enfermagem em clientes com alterações hematológicas: uso da Taxonomia I da NANDA. Rev Latino-am Enfermagem [Internet]. 1997 [cited 2009 feb 16];5(4):91-9. Available from: http://www.scielo. br/pdf/rlae/v5n4/v5n4a12. pdf.

5. Souza AM. S.O.S. Dinâmica de grupo. 1st ed. Fortaleza: Gráfica LCR; 1999.

6. McCloskey JC, Bulechek GM. Classificação das intervenções de enfermagem. 4th ed. Porto Alegre: Artmed; 2008.

7. Nogueira LCL. Gerenciando pela qualidade total na saúde. 3rd ed. Belo Horizonte: Desenvolvimento Gerencial; 2003.

8. Ortega ETT, Kojo TK, Lima DH, Veran MP, Neves MI. Compêndio de enfermagem em transplante de células tronco hematopoéticas: rotinas e procedimentos em cuidados essenciais e em complicações. 1st ed. Curitiba: Editora Maio; 2004.

9. Bonassa EMA, Santana TR. Enfermagem em terapêutica oncológica. 3rd ed. São Paulo: Atheneu; 2005.

10. Eastridge BJ, Lefor AT. Complications of indwelling venous access devices in câncer patients. J Clin Oncol. 1995; 13(1):233-8.

11. Brown-Smith JK, Stoner MH, Barley ZA. Tunneled catheter thrombosis: factors related to incidence. Oncol Nurs Forum. 1990; 17(4): 543-9.

12. Centers for Disease Control and Prevention. Guidelines for the prevention of intravascular catheter-related infections. Recommendations and Reports [Internet]. 2002 [cited 2009 mar 10]; 51(RR10): 1-29. Available from: http://www.cdc.gov/mmwr/preview/mmwrhtml/rr511 0a1.htm.

13. Silveira RCCP, Galvão CM. O cuidado de enfermgem e o cateter de Hickman: a busca de evidências. Acta paul. enferm. [Internet]. 2005 [cited 2009 feb 16];18(3):276-84. Available from: http://www.scielo.br/pdf/ape/v18n3/a08v18n3.pdf. 14. Andrade $M$, Silva SR. Administração de quimioterápicos: uma proposta de protocolo de enfermagem. Rev Bras Enferm [Internet]. 2007 [cited 2009 feb 16];60(3):331-5. Available from: http://www.scielo.br/pdf/reben/v60n3/a16.pdf. 
15. Fonseca SM, Almeida, EPM, Massunaga VM. Protocolo de intervenções de enfermagem frente às reações adversas aos quimioterápicos antineoplásicos. In: Fonseca SM, Machado RCL, Paiva DRS, Almeida EPM, Massunaga VM, Junior WR, et al. Manual de quimioterapia antineoplásica. Rio de Janeiro: Reichmann \& Affonso; 2000. p.28-54.

16. Vasques $\mathrm{Cl}$, Rodrigues CC, Reis PED, Carvalho EC. Assistência de enfermagem a portadores de linfoma de hodgkin submetidos a quimioterapia: revisão integrativa. Online braz j nurs [Internet]. 2007 [cited 2009 feb 16];7(1). Available from: http://www. uff.br/objnursing/index. php/nursing/artic le/view/j.1676-4285.2008.1416/307.

Artigo recebido em 20.11.07.

Aprovado para publicação em 31.03.09. 